\title{
Adaptação de Alunos de Medicina em Anos Iniciais da Formação
}

\section{Medical Students' Adaptation in the Early Years of College}

\author{
Márcia Miki Tanaka \\ Larissa Lazzarini Furlan ${ }^{I}$ \\ Leda Maria Branco \\ Nelson Iguimar Valerio $^{I}$
}

\section{PALAVRAS-CHAVE}

- Estudantes de Medicina;

- Saúde Mental;

- Escolha da profissão;

- Educação Médica.

\section{KEYWORDS}

- Medical Students;

- Mental Health;

- Career Choice;

- Medical Education;
Recebido em: 01/04/2015

Aprovado em: 16/05/2015

\section{RESUMO}

No início e no decorrer da graduação, problemas de adaptação, competitividade, decepção, solidão e dificuldade nos relacionamentos constituem importantes fontes de sofrimento para os graduandos de Medicina. Este estudo tem como objetivo descrever o perfil sociodemográfico e percepções de acadêmicos do segundo ano de Medicina de uma faculdade do interior do Estado de São Paulo quanto a motivações na escolha do curso e instituição, dificuldades de adaptação à vida universitária e saída de casa. Foram incluídos 38 alunos, que responderam a um questionário semiestruturado, elaborado pelos pesquisadores, que continha dados de identificação, levantamento socioeconômico e perguntas sobre o curso, disciplinas, faculdade e vida fora de casa. Nos resultados, quanto à motivação para escolha do curso, destaca-se a afinidade pela área da saúde $(n=27)$. As principais dificuldades de adaptação à graduação citadas foram excessivo número de atividades $(n=24)$ e falta de organização pessoal $(n$ =17). Em relação à saída de casa, relataram saudade do convívio familiar $(n=35)$, associada a maior liberdade $(n=19)$. Com relação à faculdade, destaca-se a falta de assistência/organização para receber os alunos $(n=11)$, sugerindo que se dê maior atenção ao acolhimento e apoio no período de adaptação.

\section{ABSTRACT}

Significant sources of suffering for medical students at the beginning and during the degree course include adaptation problems, competitiveness, disappointment, loneliness, and difficulties in relationship. This study aims to describe the socio-demographic profiles and perceptions of second-year students at a medical school in the state of São Paulo in terms of their reasons for selecting the course and institution and difficulties in adapting to university life and leaving home. We interviewed 38 students who answered a semi-structured questionnaire developed by the researchers containing identification data, a socio-economic survey, as well as questions on the course, subjects, college, and their lives away from home. The results reveal that the main motivation for choosing the course was an affinity for the field of healthcare $(n=27)$. The main difficulties in adapting to the course were due to the excessive number of activities $(n=24)$ and lack of personal organization $(n=17)$. Regarding leaving home, $(n=35)$ reported that they miss their families, associated with greater freedom $(n=$ 19). With regard to college, $(n=11)$ stated that there is a lack of support/organization for students, suggesting greater attention and support are needed in the adaptation period. 


\section{INTRODUÇÃO}

Na década de 1950, foram realizadas conferências na Europa com o objetivo de discutir a saúde mental e dificuldades psicológicas do estudante de Medicina ${ }^{1}$. Inicialmente, supunha-se que a satisfação do aluno estava associada com aprovação em um dos vestibulares mais concorridos, alcançar o sonho de descobrir o corpo humano e cursar Medicina, profissão idealizada pela população².

Contudo, diversos estudos demonstram que, quando as expectativas em relação à universidade não se concretizam, surgem quadros de depressão ${ }^{3}$, angústia ${ }^{4}$, incerteza, desencanto e frustração dos estudantes já nos primeiros momentos do curso². As causas desses quadros podem inclusive levar a transtornos mentais mais graves ${ }^{5}$, que muitas vezes não conseguem ser enfrentados sozinhos pelos alunos, que procuram ajuda especializada ${ }^{6}$.

Segundo um levantamento da Retaguarda Emocional para o Aluno de Medicina da Santa Casa de São Paulo (Repam), os momentos mais críticos enfrentados são a entrada no curso (que requer adaptação ao novo estilo de vida), o contato com o paciente (que exige do aluno não apenas conhecimentos, mas habilidades para relacionamento interpessoal e para lidar com situações difíceis), o internato e a escolha da especialidade médica7 ${ }^{7}$. Além dos problemas pessoais a que todos estão sujeitos, o estudante enfrenta uma formação exaustiva e exigente, na qual deve internalizar inúmeros conhecimentos em pouco tempo e aprender a lidar com a dor, o sofrimento, a morte e com o contexto competitivo de sua formação. Nessa nova vida há o contato com pessoas diferentes, a ameaça do trote $^{8}$, festas, bebidas e drogas disponíveis e, algumas vezes, impostas ${ }^{9}$. Muitas vezes, as primeiras decepções ocorrem no contato com as disciplinas básicas, percebidas frequentemente como um adiamento do ingresso real na carreira escolhida ${ }^{10}$.

Uma pesquisa realizada com alunos do curso de Medicina de Botucatu, no interior do Estado de São Paulo, teve como finalidade estimar a prevalência de transtornos mentais entre os estudantes e respectivos fatores de risco. Os dados mostraram que a prevalência desses transtornos foi elevada e era ligada a experiências emocionalmente tensas, como o contato com pacientes graves e a formação de grupos. Diante dos resultados, o pesquisador sugere que as instituições formadoras estabeleçam intervenções voltadas ao acolhimento e ao cuidado com o sofrimento dos estudantes, visando à melhoria da qualidade de vida e à redução do sofrimento psíquico ${ }^{11}$.

Um estudo sobre a qualidade de vida e as estratégias de enfrentamento de estresse de acadêmicos de Medicina da Universidade Federal de Medicina de Santa Catarina encontrou entre estas estratégias: trabalhar a própria personalidade, resgatar hábitos de vida saudáveis e buscar a religião. A pesquisa mostrou que o não desenvolvimento de estratégias para lidar com o estresse acarretou graves consequências sobre a saúde dos entrevistados, pois os alunos disseram não ter vontade de estudar e de fazer coisas que antes eram prazerosas para eles. Os autores concluíram que a maioria das intervenções desenhadas para reduzir o estresse na educação médica se mostrou efetiva e que praticamente todos os participantes consideraram os programas úteis; que a escola médica deve estar preparada para receber, escutar, respeitar e ajudar o estudante a desenvolver estratégias para lidar com a pressão que vivenciará no cotidiano acadêmico e profissional; deve também fornecer suporte psicológico e pedagógico de forma geral e em especial aos que passam por dificuldades ${ }^{12}$.

$\mathrm{O}$ artigo "A formação da identidade do médico: implicações para o ensino de graduação em Medicina", de Ana Teresa de Abreu Ramos Cerqueira, discute a constituição da identidade do médico, tendo como pontos de partida sua escolha e formação profissionais. Esse artigo ressalta que as escolas médicas e seus professores devem ter conhecimento das dificuldades enfrentadas pelos estudantes de Medicina relacionadas não apenas com questões curriculares e pedagógicas, mas também com a formação da identidade médica. Durante a formação, é necessário refletir sobre o ser humano que há no médico, e, neste sentido, a escola tem também o papel de ajudar o aluno a entender e se adaptar a sua nova situação ${ }^{13}$.

Tomando por base as considerações anteriores, o presente estudo mobilizou questionamentos a respeito da adaptação dos jovens à vida universitária, como estão sendo recebidos, acolhidos e orientados no início da vida acadêmica. Este trabalho tem como objetivo focalizar os principais problemas enfrentados pelos alunos no início de sua formação, incluindo a entrada no curso e as dificuldades decorrentes, e como o aluno considera sua adaptação ao início do curso superior.

\section{CASUÍSTICA E MÉTODOS}

Participaram do estudo alunos que cursavam o segundo ano do curso de Medicina da Faculdade de Medicina de São José do Rio Preto (Famerp), em maio de 2013. Os estudantes foram abordados durante o horário de aula, em classe, após anuência do professor da disciplina ministrada, e convidados a participar do estudo. Após terem sido esclarecidos sobre a pesquisa, aqueles que concordaram em participar ( $\mathrm{n}=38$ de um universo composto de 64 graduandos) assinaram o Termo de Consentimento Livre e Pós-Esclarecido, elaborado em conformidade com os princípios da Resolução 196/96 do Conselho Nacional de Saúde (CNS) do Ministério da Saúde. O projeto foi aprovado pelo Comitê de Ética em Pesquisa da Famerp de acordo com o parecer 17177413.0.0000.5415. 
Os participantes responderam individualmente a um questionário semiestruturado com 36 perguntas (25 fechadas, em forma de múltipla escolha, e 11 abertas) que continham dados de identificação sociodemográficos, com questões sobre idade, sexo, religião, estado civil, região de origem e renda mensal média, e enquetes sobre os motivos que os levaram à escolha do curso, adaptação do aluno nos anos iniciais da graduação e na saída de casa, e situações angustiantes e gratificantes vividas nesse período. Esse questionário foi dividido em três partes, para melhor caracterização: Sobre o curso, disciplinas e faculdade (questões de 1 a 20); Sobre a vida fora de casa (questões de 21 a 31); Sobre as experiências de "bixo" e mudança de "bixo" para veterano (questões de 32 a 36).

Foram critérios de inclusão: estar regularmente matriculado no semestre letivo correspondente ao período de coleta dos dados e estar presente na aula no momento da coleta dos dados.

Os dados foram agrupados por categorias de respostas com significados semelhantes, com base no julgamento de juízes interdependentes, com Índice de Concordância (IC) igual ou maior que $75 \%$ (IC = A/A + D.100, onde A é igual a acordos, e D a desacordos).

\section{RESULTADOS E DISCUSSÃO}

\section{Caracterização da amostra}

Dos 38 graduandos do segundo ano de Medicina participantes, a maioria era do sexo feminino ( $n=26,68,4 \%$ ), com idade média de 21 anos, solteiros $(n=37,97,3 \%)$, católicos $(n=17$, $44,7 \%$ ), procedentes do interior do Estado de São Paulo (n = $22,57,8 \%$ ). A renda mensal média prevaleceu entre 10 e 30 salários mínimos ( $\mathrm{n}=16,42,1 \%$ ) (Tabela 1$)$.

\begin{tabular}{|c|c|c|}
\hline \multicolumn{3}{|c|}{ TABELA 1} \\
\hline & Frequência & Porcentagem \\
\hline \multicolumn{3}{|l|}{ Sexo } \\
\hline Feminino & 26 & 68,42 \\
\hline Masculino & 12 & 31,58 \\
\hline \multicolumn{3}{|l|}{ Idade (anos) } \\
\hline Menor idade $=18$ & 1 & \\
\hline Maior idade = 35 & 1 & \\
\hline Média = 21,15 & & \\
\hline \multicolumn{3}{|l|}{ Estado civil } \\
\hline Solteiro & 37 & 97,36 \\
\hline Separado & 1 & 2,64 \\
\hline \multicolumn{3}{|l|}{ Religião } \\
\hline Católico & 17 & 44,74 \\
\hline Espírita & 7 & 18,42 \\
\hline Evangélico & 4 & 10,53 \\
\hline
\end{tabular}

\begin{tabular}{|c|c|c|}
\hline \multicolumn{3}{|c|}{$\begin{array}{c}\text { TABELA } 1 \\
\text { Descrição dos dados sociodemográficos }\end{array}$} \\
\hline & Frequência & Porcentagem \\
\hline Ateu & 3 & 7,89 \\
\hline Sem religião & 3 & 7,89 \\
\hline Sem resposta & 2 & 5,27 \\
\hline Judeu & 1 & 2,63 \\
\hline Deísta & 1 & 2,63 \\
\hline \multicolumn{3}{|l|}{ Região de origem } \\
\hline Grande São Paulo & 9 & 23,68 \\
\hline Interior de São Paulo & 22 & 57,89 \\
\hline São José do Rio Preto & 2 & 5,27 \\
\hline Outros Estados & 3 & 7,89 \\
\hline Sem resposta & 2 & 5,27 \\
\hline \multicolumn{3}{|l|}{ Renda familiar } \\
\hline Até 1 salário mínimo & 0 & 0 \\
\hline De 1 a 2 salários mínimos & 2 & 5,26 \\
\hline De 2 a 5 salários mínimos & 4 & 10,53 \\
\hline De 5 a 10 salários mínimos & 5 & 13,17 \\
\hline De 10 a 30 salários mínimos & 16 & 42,10 \\
\hline De 30 a 50 salários mínimos & 2 & 5,26 \\
\hline Mais de 50 salários mínimos & 2 & 5,26 \\
\hline Sem resposta & 7 & 18,42 \\
\hline
\end{tabular}

\section{Sobre a adaptação ao curso, disciplinas e faculdade}

Quando questionados sobre a motivação para a escolha do curso, a maioria dos alunos $(\mathrm{n}=27,71 \%)$ relatou afinidade com a área de saúde, 18 (47,3\%) citaram o desejo de ajudar as pessoas, e 4 (10,5\%) o status profissional/boa remuneração. Tais achados são condizentes com a literatura, que destaca a identificação pessoal, o desejo de ajudar, a busca da independência financeira e de status profissional, e a influência familiar como fatores que determinaram a decisão ${ }^{10}$.

Dos alunos analisados que cursam Medicina na Famerp, apenas $13(34,2 \%)$ optaram pela instituição como primeira opção. O principal motivo apresentado foi a distância em relação à cidade de origem, embora a maior parcela dos estudantes seja do interior de São Paulo ( $\mathrm{n}=22$ 57, 89\%). A qualidade da faculdade ( $\mathrm{n}=4,10,52 \%$ ) foi citada como fator para escolha da instituição. Segundo Kotler e Fox, citados no trabalho de Cintia Coelho Dias, o estudante prioriza as instituições de ensino com características como excelência acadêmica, convívio e localização ${ }^{14}$.

$\mathrm{O}$ estudo de Hossler aponta que os fatores determinantes na escolha do curso e da instituição são as expectativas e o encorajamento dos pais; realização acadêmica; nível de escolaridade dos pais; influência dos pares; envolvimento nas atividades escolares ${ }^{15}$. Os resultados encontrados na pesquisa 
apontam dúvidas na escolha muito parecidas com as dos demais estudantes (Tabela 2).

\begin{tabular}{|lcc|}
\hline \multicolumn{3}{c|}{ TABELA 2 } \\
Motivação em cursar medicina \\
\hline \multicolumn{1}{|c|}{ Motivações } & Frequência & Porcentagem \\
\hline Afinidade com a área da saúde & 27 & 71,05 \\
\hline Desejo de ajudar as pessoas & 18 & 47,36 \\
\hline Status profissional & 2 & 5,26 \\
\hline Boa remuneração & 2 & 5,26 \\
\hline Pais e Parentes & 1 & 2,63 \\
\hline
\end{tabular}

Embora a maioria dos alunos $(\mathrm{n}=32,84,2 \%)$ tenha se declarado satisfeita com a escolha, períodos de insatisfação, frustração e desejo de mudança são comuns. Esses dados são compatíveis com a literatura:

Os momentos vividos no decorrer do curso de Medicina nem sempre são gratificantes, e a fase inicial de euforia do aluno é substituída por uma fase posterior de desencanto, com queixas frequentes, como excessivo volume de estudos e tempo escasso, aulas monótonas e professores desatualizados(... ${ }^{16}$ (p.18)

Os achados parecem corroborar a ideia de ciclo ${ }^{17}$, em que o ajustamento psicológico à escolha vai sofrendo alterações à medida que o aluno vai passando por diferentes experiências e momentos de transição, como o ingresso no ensino superior ou a inserção no mercado de trabalho ${ }^{18}$. O período intermediário da formação é considerado um momento de calmaria ou acomodação no que se refere às questões vocacionais ${ }^{19}$.

O primeiro ano da graduação no curso superior é um período crítico na vida acadêmica, pois exige adaptação e integração ao novo ambiente, influenciando o restante da formação do futuro profissional ${ }^{20}$. Assim, a transição para o curso superior coloca em evidência os problemas dos alunos e em alguns casos pode inclusive exacerbá-los.

Entre os problemas enfrentados, a maioria dos avaliados no presente estudo $(n=24,63,1 \%)$ julgou que o número de atividades a ser realizada em curto espaço de tempo e a falta de organização pessoal ( $\mathrm{n}=17,44,7 \%$ ) são os fatores que mais dificultam a adaptação à graduação.

Quando indagados sobre as dificuldades no curso superior, a maioria ( $n=12,31,5 \%$ ) relatou falta de assistência/organização da faculdade em receber os alunos, adaptação ao novo ritmo de vida ( $\mathrm{n}=8,21 \%$ ) e distância de casa $(\mathrm{n}=7,18,4 \%)$. A literatura apresenta como problemas enfrentados pelos alunos: solidão, timidez, limitações nas competências sociais e tomadas de decisão, sexualidade e perturbações emocionais, convívio com os novos colegas ${ }^{21}$, dificuldade de relacionamento com professores e colegas, nova rotina, cobrança nos estudos, baixo rendimento escolar, dificuldade para administrar o tempo, ansiedade, estresse nas avaliações e falta de informação por parte da instituição. Acrescentam-se ainda problemas financeiros, de gestão da moradia, de acomodação, de hábitos alimentares e de segurança ${ }^{22}$ (Tabela 3 ).

\begin{tabular}{|lcc|}
\multicolumn{3}{|c|}{ TABELA 3} \\
Dificuldades em relação à Faculdade \\
\hline \multicolumn{1}{|c|}{ Dificuldades } & Frequência & Porcentagem \\
\hline $\begin{array}{l}\text { Falta de assistência/organização em } \\
\text { receber os alunos }\end{array}$ & 12 & 31,57 \\
\hline Novo ritmo de vida & 8 & 21,05 \\
\hline Sem resposta & 8 & 21,05 \\
\hline Distância de casa & 7 & 18,42 \\
\hline Nenhuma & 4 & 10,52 \\
\hline
\end{tabular}

Quanto à graduação, os alunos relataram maiores dificuldades em relação ao grande número de atividades a serem executadas $(n=24,63,1 \%)$ e à falta de organização pessoal (n $=17,44,7 \%$ ). Pesquisas realizadas em faculdades de Medicina revelaram como fontes de estresse os exames e as provas, grande quantidade de material a ser estudado, falta de tempo para familiares, amigos e lazer ${ }^{23,27}$, problemas semelhantes aos apresentados pelos estudantes analisados.

Os dados encontrados na pesquisa são condizentes com a literatura, revelando dificuldade de adaptação à vida universitária durante o primeiro ano do curso, porém atingindo índices de satisfação em torno de $80 \%$ no segundo ano, revelando uma mudança na maneira de encarar os problemas. Nesse momento do curso, começa a aumentar a preocupação com os estudos e a formação profissional, enquanto a saudade dos familiares permanece ${ }^{17}$.

\section{Sobre a vida fora de casa}

A maioria dos estudantes da pesquisa mora longe da família (n $=36,94,7 \%$ ). Deixar a casa dos pais e a família é um evento marcante e pode ser encarado como uma dificuldade, pois o aluno sente saudade e solidão, e também como uma conquista de independência e liberdade ${ }^{24}$. A distância do local da residência é um fator importante para desenvolver saudade, pois os estudantes que se mudam para um lugar distante de casa procuram feriados, finais de semana e férias para regressar e apresentam maior tendência a se isolar do meio acadêmico e das atividades extracurriculares ${ }^{22}$. Este estudo verificou que a saudade é muito 
marcante na vida dos estudantes nos anos iniciais da graduação ( $\mathrm{n}=35,92,1 \%)$, mostrando que, apesar da nova vida, os alunos ainda dependem do apoio e proximidade dos familiares/ pessoas a que estavam acostumados, embora citem a sensação de maior liberdade associada ( $n=19,50 \%)$ (Tabela 4$)$.

\begin{tabular}{|lcc|}
\hline \multicolumn{4}{c|}{$\begin{array}{c}\text { TABELA } \\
\text { Sentimentos ao viver longe de casa }\end{array}$} \\
\hline \multicolumn{1}{|c|}{ Sentimento } & Frequência & Porcentagem \\
\hline Falta da família & 22 & 57,89 \\
\hline Maior liberdade & 19 & 50 \\
\hline Falta dos amigos & 12 & 31,57 \\
\hline Falta do(a) namorado(a) & 8 & 21,05 \\
\hline Sem resposta & 4 & 10,52 \\
\hline Solidão & 2 & 5,26 \\
\hline
\end{tabular}

Os dados apontaram ainda que, diante de algum problema pessoal, os pais e familiares são os primeiros a quem o estudante recorre $(n=23,60,5 \%)$, seguidos do melhor amigo ( $\mathrm{n}=$ $15,39,4 \%)$ e namorado(a) $(n=10,26,3 \%)$, pois são pessoas nas quais confiam, consideradas pontos de apoio. Um dado interessante foi que, entre os entrevistados, nenhum relatou procurar ajuda de psicólogos, tutores ou professores da instituição; porém, mesmo assim, consideraram essencial a existência de um serviço de apoio sociopsicológico por acreditarem que muitas dessas dificuldades não conseguem ser resolvidas individualmente, requerendo a ajuda de um profissional (Tabela 5).

\begin{tabular}{|lcc|}
\hline \multicolumn{3}{c|}{ TABELA 5 } \\
Descrição sobre o auxílio procurado \\
$\quad$ em momentos de dificuldade \\
\hline Pessoa procurada & Frequência & Porcentagem \\
\hline Pais e Familiares & 23 & 60,52 \\
\hline Melhor amigo & 15 & 39,47 \\
\hline Namorado (a) & 10 & 26,31 \\
\hline Deus & 1 & 2,63 \\
\hline Não recorre & 1 & 2,63 \\
\hline Sem resposta & 1 & 2,63 \\
\hline Professores, psicólogos, tutores & 0 & 0 \\
\hline
\end{tabular}

\section{Sobre as experiências vividas no trote}

Em relação à experiência como ingressante do curso, os alunos se mostraram divididos quanto à existência e importância do trote na vida acadêmica. A maioria $(n=22,57,8 \%$ ) considerou o trote como não importante, e 16 alunos (42,1\%) acreditam se tratar de um rito de passagem marcante e importante na vida. Em relação ao impacto do trote, os alunos relataram tanto afastamento em relação aos veteranos ( $\mathrm{n}=11,28,9 \%)$, quanto aproximação dos veteranos e colegas de turma $(n=10,26,3 \%)$. Ao serem indagados, a maioria ( $n=21,55,2 \%$ ) declarou julgar interessante estabelecer uma forma de aprendizagem sem agressividade.

O trote admitido como brincadeira é considerado um ritual de passagem para o curso superior de grande importância, que marca a nova situação do calouro ${ }^{25}$ e uma forma de integração à vida universitária, desde que mantenha a integridade do ingressante ${ }^{26}$.

\section{CONCLUSÃO}

O início da vida no ensino superior é desafiador para o estudante, que passa por inúmeras mudanças, de ambiente estudantil, muitas vezes de cidade, com novos colegas, distante de casa e familiares. É um momento de grande estresse psicológico, decorrente de todas as adaptações que deverão ocorrer nessa nova fase.

Espera-se que os dados obtidos neste estudo contribuam para maior atenção às dificuldades e às questões referentes ao momento de início e adaptação no nível superior. Deseja-se ainda que se implantem formas mais eficazes de apoio sociopsicológico e programas de intervenções para auxiliar os futuros médicos a lidar com a profissão, a saúde e seus pacientes.

\section{REFERÊNCIAS}

1. Pacheco e Silva AC, Lipszic SL. Estudantes de Medicina hoje. São Paulo: Universidade de São Paulo; 1962.

2. Dini OS, Batista NA. Graduação e prática médica: expectativas e concepções de estudantes de Medicina do $1^{\circ}$ ao $6^{\circ}$ ano. Rev Bras Educ Med. 2004; 28(3):198-203.

3. Vallilo NG, Júnior RD, Gobbo R, Novo NF, Hübner CVK. Prevalência de sintomas depressivos em estudantes de Medicina. Rev Bras Clin Med. 2011; 9(1):36-41.

4. Quintana AM, Rodrigues AT, Arpini DM, Santos MS. A angústia na formação do estudante de Medicina.Rev. bras. educ. med. 2008; 32(1):7-14.

5. Almeida AM, Godinho TM, Bitencourt AGV, Teles MS, Silva AS, Fonseca DC, et al. Common mental disorders among medical students.J. bras. psiquiatr. 2007; 56(4):245-251.

6. Millan LR, De Arruda PCV. Assistência psicológica ao estudante de Medicina: 21 anos de experiência.Rev. Assoc. Med. Bras. 2008; 54(1): 90-94.

7. Bellodi PL. Retaguarda Emocional Para o Aluno de Medicina da Santa Casa de São Paulo (REPAM): realizações e reflexões.Rev. bras. educ. med. 2007; 31(1):5-14.

8. Marin JC, Araújo DCS, Neto JE. O trote em uma faculdade de Medicina: uma análise de seus excessos e influências socioeconômicas. Revista Brasileira de Educação Médica. 2008; 32(4)474-481. 
9. Lemos KM, Neves NMBC, Kuwano AY, Tedesqui G, Bitencourt AGV, Neves FBCS, et al . Uso de substâncias psicoativas entre estudantes de Medicina de Salvador (BA). Rev. psiq. clín. 2007; 34(3):118-124.

10. Trindade LMDF, Vieira MJ. Curso de Medicina: motivações e expectativas de estudantes iniciantes. Rev. bras. educ. med. 2009; 33(4): 542-554.

11. Lima MCP, Domingues MS, Cerqueira ATAR. Prevalência e fatores de risco para transtornos mentais comuns entre estudantes de Medicina. Rev. Saúde Pública. 2006; 40(6):1035-1041.

12. Zonta R, Robles ACC, Grosseman S. Estratégias de enfrentamento do estresse desenvolvidas por estudantes de Medicina da Universidade Federal de Santa Catarina.Rev. bras. educ. med. 2006; 30(3):147-153.

13. Ramos-Cerqueira ATA, Lima MCP. A formação da identidade do médico: implicações para o ensino de graduação em Medicina.Interface (Botucatu). 2002; 6(11):107-116.

14. Dias CC. Os fatores de influência no processo decisório do aluno universitário. Vila Velha: Centro Universitário de Vila Velha; 2004.

15. Hossler D, Schmit J, Vesper, N. Going to College: how social, economic, and educational factors influence the decisions students make. Baltimore: The Johns Hopkins University Press; 1999.

16. Moreira SNT, Silva CAN, Tertulino FF, Tertulino FMF, Vilar MJP, Azevedo GD. Processo de significação de estudantes do curso de Medicina diante da escolha profissional e das experiências vividas no cotidiano acadêmico. Rev. bras. educ. med. 2006;30(2):14-19.

17. Lassance MCP. A orientação profissional e a globalização da economia. Rev. Abop. 1997; 1(1):71-80.

18. Kalakoski V, Nurmi JE. Identity and educational transitions: age differences in adolescent exploration and commitment related to education, occupation and family. Journal of Research on Adolescence. 1998; 8(1):29-47.

19. Bardagi MP, Lassance MCP, Paradiso AC. Trajetória Acadêmica e Satisfação com a Escolha Profissional de Universitários em Meio de Curso. Rev. bras. orientac. Prof. 2003; 4(1-2):153-166.

20. Cunha SM, Carrilho DM. O processo de adaptação ao ensino superior e o rendimento acadêmico.Psicol. Esc. e Educ. 2005; 9(2):215-224.

21. Ferraz MF, Pereira AS. A dinâmica da personalidade e o homesickness (saudades de casa) dos jovens estudantes universitários.Psic., Saúde \& Doenças. 2002; 3(2):149-164.

22. Cechet AGS. O ingresso na universidade pública: Análise dos sentidos atribuídos por um grupo de estudantes aten- didos pelas assistência estudantil. Curitiba; 2013. Mestrado [Dissertação] - Universidade Federal do Paraná.

23. Furtado ES, Falcone EMO, Clarck C. Avaliação do estresse e das habilidades sociais na experiência acadêmica de estudantes de Medicina de uma universidade do Rio de Janeiro. Interac. em psicol. 2003; 7(2):43-51.

24. Teixeira MAP, Dias ACG, Wottrich SH, Oliveira AM. Adaptação à universidade em jovens calouros.Psicol. Esc. Educ. 2008; 12(1): 185-202.

25. Warth MPTN, Lisboa LF. Tradição, trote e violência. Interface (Botucatu). Botucatu. 1999;3(5):111-118.

26. Giarola LC. Trote na universidade. Interface (Botucatu). Botucatu. 1999;3(5):127-128.

27. Neves N, Lemos K, Bitencourt A, Neves FS, Nunes C, Neville I, Kuwano A, Duraes L. Expectativa versus Realidade na Formação Médica: o (Des)encanto do Estudante de Medicina na formação. Gaz. Méd. 2006;76(2):20-28.

\section{CONTRIBUIÇÃO DOS AUTORES}

Márcia Miki Tanaka: Graduanda da faculdade de medicina. Participou da elaboração do projeto e dos questionários, aplicação dos mesmos, e análise dos dados coletados e elaboração do artigo.

Larissa Lazzarini Furlan: Graduanda da faculdade de medicina. Participou da elaboração do projeto e dos questionários, aplicação dos mesmos, e análise dos dados coletados e elaboração do artigo.

Leda Maria Branco: Psicóloga do Departamento de Psiquiatria e Psicologia. Participou da elaboração do projeto e dos questionários, aplicação dos mesmos, análise dos dados coletados e elaboração do artigo. Supervisionou a elaboração do projeto e dos questionários, a aplicação dos mesmos aos estudantes e a elaboração do artigo.

Nelson Iguimar Valério: Mestre e Doutor em Psicologia. Participou como orientador na elaboração do projeto e dos questionários, aplicação dos mesmos, e análise dos dados coletados e elaboração do artigo. Supervisionou a elaboração do projeto e dos questionários, a aplicação dos mesmos aos estudantes e a elaboração do artigo.

\section{CONFLITO DE INTERESSES}

Os autores declaram não haver conflito de interesses.

\section{ENDEREÇO PARA CORRESPONDÊNCIA}

Márcia Miki Tanaka

Rua Dr. Francisco Castro, 194 - apto 21

Bosque da Saúde - São José do Rio Preto

CEP: 15091-170 SP

E-mail: mmt_marciamt@hotmail.com 